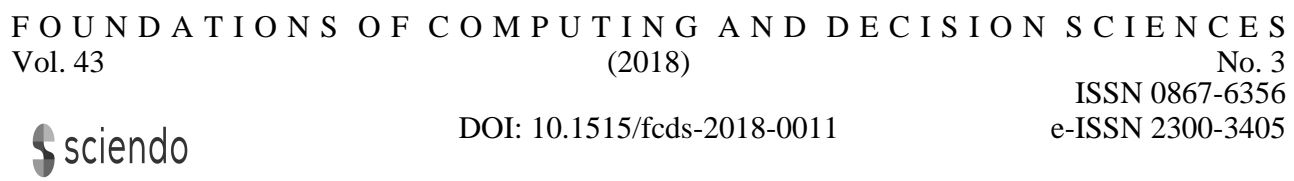

\title{
Supervised Machine Learning with Control Variates for American Option Pricing
}

\author{
Gang $\mathrm{Mu}{ }^{*} \dagger$, Teodor Godina ${ }^{\dagger}$, Antonio Maffia ${ }^{\dagger}$, Yong Chao Sun *
}

\begin{abstract}
In this paper, we make use of a Bayesian (supervised learning) approach in pricing American options via Monte Carlo simulations. We first present Gaussian process regression (Kriging) approach for American options pricing and compare its performance in estimating the continuation value with the Longstaff and Schwartz algorithm. Secondly, we explore the control variates technique in combination with Kriging to further improve the estimation of the continuation value. This method allows to reduce dramatically the standard errors and to improve the stability of the Kriging approach. For illustrative purposes, we use American put options on a stock whose dynamics is given by Heston model, and use European options on the same stock as control variates.
\end{abstract}

Key Words: American options, Monte Carlo, Gaussian processes, Kriging, LSM, supervised learning, Heston Model, control variates

\section{Introduction}

American options pricing efficiency remains a topic of heated interest and research in Computational Mathematics and Finance. American style options offer great flexibility in all financial and trading markets such as stock, equity, commodity, credit and forex due to the possibility of an early exercise. However, there are no closedform analytical valuation of these types of derivatives because of the optimal exercise problem created by the early exercise.

The increasing difficulty of pricing American options when the underlying follows a complex stochastic process dynamics have turned researchers to the use of the Monte Carlo simulations. Monte-Carlo simulations complexity increases linearly with

\footnotetext{
* School of Mathematical Sciences, Tongji University, 200092, Shanghai, China

${ }^{\dagger}$ F. Hoffmann-La Roche AG, Grenzacherstrasse 124, 4070 Basel, Switzerland

$\ddagger$ Department of Mathematics and Computer Science, University of Basel
} 
the dimension of the problem, whereas methods like finite difference's complexity is exponential. The Monte Carlo approach to price American options is accompanied with the question on how to estimate the continuation value and execute the optimal exercise. Most of the developed methods in this area are related to supervised learning i.e. estimating ' $\mathbb{E}[Y \mid X]=f(X)+\epsilon$ ' via parametric/non-parametric techniques. Many researcher attempted to solve the problem such as Tsitsiklis and Van Roy [15] with parametric regression, Pizzi and Pellizzari [11] with Nadaraya-Watson kernel regression and Li, Szepesvari and Schuurmans [7] with LSPI algorithm. A major breakthrough in pricing American options with Monte Carlo approach was developed by Longstaff and Schwartz [8] and it is considered a "golden standard" in the industry and literature. Longstaff and Schwartz method (LSM) uses parametric regression onto basis functions. In this paper, the authors have proved that when increasing the number of Monte-Carlo simulations and the basis functions (e.g. polynomial type basis function) the LSM method converges. However, it has slow convergence rate and the computational time increases exponentially as long as the number of basis functions and dimension complexity increase.

In this paper, we review a supervised learning approach namely Gaussian process regression (GPR) which was used the first time by Ludkovski [10] in estimating the continuation value of American style options. Gaussian process regression, also named Kriging, with squared exponential kernel requires only one basis function per dimension to be able to learn the continuation value, creating a smoother exercise boundary. We aim to improve it with the application of control variate and give Kriging more flexibility in pricing American options over the LSM methodology on multidimensional stochastic processes. Also, this will allow Kriging method to learn the exercise policies from much smaller sample and can be applied to the rest of the paths, 'sub-sample strategy' Gramacy and Ludkovski [5].

Our result are mostly focus on the Heston model, a 2-dimensional process, to show the efficiency of the Kriging with control variate over the LSM method. Heston model offers more flexibility in pricing options on the stock market due to its stochastic volatility which can replicate the implied volatility smile observed of the options traded in financial markets.

The paper is structured as follows. In the section 2, we review the backward dynamics principle, LSM approach and Kriging algorithm. We explain how to apply the control variates technique with the aid of the GPR. Section 3 contains our numerical analysis where we test our approach on synthetic and market data. Finally, we summarize our findings in the section 4.

\section{Pricing American Options}

We assume that the financial market has an underlying complete probability space $(\Omega, \mathcal{F}, \mathbb{P})$, a filtration $\left\{\mathcal{F}_{t}\right\}_{0 \leq t \leq T}$ with finite time horizon $[0, T]$ and $\mathcal{F}_{T}=\mathcal{F}$. Under the no-arbitrage condition, we can suppose the existence of equivalent martingale measure $\mathbb{Q} \sim \mathbb{P}$. Furthermore, let $S_{t}$ be the state variable (i.e. price of the asset) and the $h\left(S_{t}\right)$ the payoff of the options restricted to the square-integrable space $\mathcal{L}^{2}(\Omega, \mathcal{F}, \mathbb{P})$. 
In the pricing framework, we will be using Heston 2-dimensional model to depart from Black-Scholes 1-dimensional dynamics and to show the performance the Kriging method on a multidimensional asset. Heston model is a mathematical model which assumes a non-constant volatility of an underlying asset and was first introduced by Heston [6]. Let $S_{t}$ be the underlying asset under risk neutral measure with variance $v_{t}$ that follows a CIR process:

$$
\begin{aligned}
d \ln S_{t} & =\left(r-\frac{1}{2} v_{t}\right) d t+\sqrt{v_{t}} d W_{1, t}^{Q} \\
d v_{t} & =\kappa\left(\theta-v_{t}\right) d t+\sigma \sqrt{v_{t}} d W_{2, t}^{Q} \\
<d W_{1, t}^{Q}, d W_{2, t}^{Q}> & =\rho d t
\end{aligned}
$$

where $S_{0} \geq 0, v_{0}>0$ are initial value of the asset and its variance and $W_{1, t}^{Q}, W_{2, t}^{Q}$ are standard Wiener process with the following parameters: $r$ - risk free rate, $|\rho|<1$ correlation of $W_{1, t}^{Q}$ and $W_{2, t}^{Q}, \kappa>0$ - mean reverting rate of variance, $\theta>0$ - long run variance, and $\sigma>0$ - volatility of variance.

American options with maturity $T$ can be formulated as a process $\left\{H_{\tau} \in \mathcal{F}_{\tau}\right\}_{0 \leq \tau \leq T}$ which represents the discounted payoff of the option in case of exercise at time $\tau$. Denote $\mathcal{T}$ of admissible stopping time $\tau$ taking values $[0, T]$. Then the initial price of this option can be written as:

$$
P_{0}=\sup _{\tau \in \mathcal{T}} \mathbb{E}\left[H_{\tau}\right]
$$

Let the financial asset $S_{\tau}$ follow Heston dynamics then an American put option with maturity $T$ and strike price $K$ written on an asset $S_{\tau}$ has payoff $h\left(S_{\tau}\right)=\left(K-S_{\tau}\right)^{+}$ and the following price at time $t$ :

$$
P(S, v, t)=\sup _{\tau \in \mathcal{T}} \mathbb{E}\left[e^{-r \tau}\left(K-S_{\tau}\right)^{+}\right]
$$

The supremum is achieved for stopping optimal time $\tau^{*}$ :

$$
\tau^{*}=\inf \left\{\tau \in \mathcal{T}: S_{\tau} \leq B_{\tau}\right\}
$$

where $B_{\tau}$ is called optimal exercise boundary.

\subsection{Monte Carlo Formulation}

In order to be able to tackle numerically the American option pricing problem with Monte Carlo method, we have to depart from the continuous case and restrict to option classes with discrete exercise. Bermudan options are a hybrid between American options and European which allows the option to be exercised at specific dates before maturity. In our case lets define uniform discretization of the time interval $[0, T]$ with distance $\Delta t=\frac{T}{N}$ and $0=t_{0}<t_{1}<\ldots<t_{N}=T$ equidistant exercise opportunities. The asset value $S_{t}$ is also simulated at the same time steps $\left\{t_{i}\right\}_{i \in\{0, . ., N\}}$ as the discretization. The simulation of $S_{t_{1}}, \ldots, S_{t_{N}}$ is affected by discretization error which is described in detail by Kloeden \& Platen [9] and Glasserman [3]. 
Monte Carlo methods for pricing American option are based on the backward dynamic programming formulation which estimates recursively the value of the option. Let's denote $X=(S, v)$ the underlying stochastic process, $h\left(X_{t_{i}}\right)$ the discounted payoff of the option, and $V_{t_{i}}$ the discounted value of the option at time $t_{i}$. The recursive estimation of the option value is defined in the following way:

$$
\begin{aligned}
V_{t_{N}} & =h\left(X_{t_{N}}\right) \\
V_{t_{i-1}} & =\max \left\{h\left(X_{t_{i-1}}\right), \mathbb{E}\left[V_{t_{i}} \mid X_{t_{i-1}}\right]\right\} \\
& i=1,2, \ldots, N
\end{aligned}
$$

The above equation states that the value of the option at expiration time $t_{N}$ is exactly the payoff of the option $h\left(X_{t_{N}}\right)$. At any other time $0 \leq t_{i}<t_{N}$, the value of the option $\bar{V}_{t_{i-1}}$ is the maximum between immediate exercise $\bar{h}\left(X_{t_{i-1}}\right)$ and the continuation value $\mathbb{E}\left[V_{t_{i}} \mid X_{t_{i-1}}\right]$ which is the discounted present value of holding the option rather than exercising it at time $t_{i}$. We denote the continuation value as:

$$
\begin{array}{ll}
\mathcal{C}_{t_{i-1}} & =\mathbb{E}\left[V_{t_{i}} \mid X_{t_{i-1}}\right] \\
& i=1,2,3, \ldots, N-1
\end{array}
$$

Since the payoff $h\left(X_{t_{N}}\right) \geq 0$ is always non-negative, we can set $\mathcal{C}_{t_{N}}=0$ i.e. at expiry it is no longer optimal to hold the option. Conversely, we obtain the value function $V_{t_{i}}$ for $i=1, \ldots, N$ :

$$
V_{t_{i}}=\max \left\{h\left(X_{t_{i}}\right), \mathcal{C}_{t_{i}}\right\}
$$

At the last step $t_{0}=0$, we estimate the option value as:

$$
\bar{V}_{0}=\frac{1}{M} \sum_{j=1}^{M} V_{t_{0}}^{(j)}
$$

where $M$ is the number of simulated paths of the Heston process $X_{t}$.

\subsection{Kriging Approach}

The continuation value $\mathcal{C}$ remain a 'Black box' and a pivotal piece in the valuation of American option. In the Monte Carlo framework, the continuation value can be viewed as following estimation problem:

$$
\begin{aligned}
V_{t_{i}} & =\tilde{\mathcal{C}}_{t_{i-1}}+\epsilon_{t_{i-1}} \\
& =f_{i-1}\left(X_{t_{i-1}}\right)+\epsilon_{t_{i-1}}
\end{aligned}
$$

where $f_{i-1}$ is a measurable function and $\epsilon_{t_{i-1}}$ is the error term with mean 0 and variance $\sigma_{t_{i-1}}$.

Longstaff and Schwartz [8] introduced an innovative parametric approach to derive the continuation value. For in the money paths, they estimate $\mathcal{C}$ via linear combination of $K$ basis function $\phi_{k}(X)$ which are dependent on the underlying stochastic process $X$ : 


$$
\tilde{\mathcal{C}}\left(X_{t_{i}}\right)=\sum_{k=1}^{K} \beta_{k, i-1} \phi_{k}\left(X_{t_{i-1}}\right)=\Phi\left(X_{t_{i-1}}\right)^{T} \beta_{i-1}
$$

with $\beta_{i-1}$ minimizing error term $\epsilon_{t_{i-1}}$ in equation (8):

$$
\left.\beta_{i-1}=\left(\mathbb{E}\left[\Phi\left(X_{t_{i-1}}\right) \Phi\left(X_{t_{i-1}}\right)^{T}\right]\right)^{-1} \mathbb{E}\left[\Phi\left(X_{t_{i-1}}\right) V_{t_{i}}\right)\right] .
$$

Although LSM is a reliable and precise algorithm, finding the sufficient number of simulations and optimal number of basis function [4] is becoming more difficult to perform with higher dimensional stochastic processes. For this purpose, we introduce a non-parametric approach Gaussian process regression.

A Gaussian process (GP) is a collection of random variables where any subset has a Gaussian distribution. A Gaussian process:

$$
f(X) \sim \mathcal{G P}\left(m(X), \mathcal{K}\left(X, X^{T}\right)\right)
$$

is only defined by the mean $m(X)$ and covariance matrix $\mathcal{K}\left(X, X^{T}\right)$ for a positive and symmetric kernel $\mathcal{K}$. There are many choices for kernel but one of most preferred kernels is the squared exponential function:

$$
\mathcal{K}(x, y)=\sigma_{f}^{2} \exp \left(-\frac{1}{2 l^{2}}\|x-y\|_{2}^{2}\right)
$$

Where the hyperparameters $\sigma_{f}$ and $l$ are found by maximizing the log-likelihood. Since this kernel is symmetric and positive, it produces positive definite and symmetric covariance matrix.

Due to the flexibility of GPR, we don't need to use all the simulated paths to estimated all the parameters. We select a random subset $X_{t_{i-1}}^{\prime}$ of the simulated paths of size $M^{\prime}$ and $V_{t_{i}}^{\prime}$ the corresponding path-wise option values. Using equation (8), we can represent the option value as a Gaussian distribution:

$$
V_{t_{i}}^{\prime} \sim \mathcal{N}\left(m\left(X_{t_{i-1}}^{\prime}\right), \mathcal{K}\left(X_{t_{i-1}}^{\prime}, X_{t_{i-1}}^{\prime T}\right)+\sigma_{t_{i-1}} I\right)
$$

We have to find the optimal hyperparameters $\eta=\left(\sigma_{f}, l, \sigma_{t_{i-1}}\right)$. For this reason we introduce the marginal likelihood $p\left(V_{t_{i}}^{\prime} \mid X_{t_{i-1}}^{\prime}\right)$ :

$$
p\left(V_{t_{i}}^{\prime} \mid X_{t_{i-1}}^{\prime}\right)=\int p\left(V_{t_{i}}^{\prime} \mid f, X_{t_{i-1}}^{\prime}\right) p\left(f \mid X_{t_{i-1}}^{\prime}\right) d f
$$

We have $V_{t_{i}}^{\prime}=f\left(X_{t_{i-1}}^{\prime}\right)+\epsilon\left(X_{t_{i-1}}^{\prime}\right)$ and $\epsilon\left(X_{t_{i-1}}^{\prime}\right) \sim \mathcal{N}\left(0, \sigma_{t_{i-1}}\right) \Rightarrow V_{t_{i}}^{\prime} \mid f\left(X_{t_{i-1}}^{\prime}\right) \sim$ $\mathcal{N}\left(f, \sigma_{t_{i-1}} I\right)$. We get prior distribution $f\left(X^{\prime}\right) \mid X^{\prime} \sim \mathcal{N}\left(m(x), \mathcal{K}\left(X^{\prime}, X^{\prime}\right)\right)$. Finally, we arrive at log-likelihood:

$$
\begin{aligned}
\log \left(p\left(V_{t_{i}}^{\prime} \mid X_{t_{i-1}}^{\prime}, \eta\right)\right)= & -\frac{1}{2} V_{t_{i}}^{\prime T}\left[\mathcal{K}\left(X_{t_{i-1}}^{\prime}, X_{t_{i-1}}^{\prime}\right)+\sigma_{t_{i-1}} I\right]^{-1} V_{t_{i}}^{\prime} \\
& -\frac{1}{2} \log \left[\mathcal{K}\left(X_{t_{i-1}}^{\prime}, X_{t_{i-1}}^{\prime}\right)+\sigma_{t_{i-1}} I\right]-\frac{M^{\prime}}{2} \log (2 \pi)
\end{aligned}
$$


By maximizing the log-likelihood in (15), we obtain the desire parameters $\eta=$ $\left(\sigma_{f}, l, \sigma_{t_{i-1}}\right)$ for the Gaussian process regression. We have divided the total number of simulations into two sets $X_{t_{i-1}}^{\prime}$ (learning set) and the complement $X_{t_{i-1}}^{*}$ (prediction set). If we apply same estimates to the prediction set we get a noise-free estimation of the continuation value for the entire set:

$$
\left[\begin{array}{l}
f\left(X_{t_{i-1}}^{\prime}\right) \\
f\left(X_{t_{i-1}}^{*}\right)
\end{array}\right] \sim \mathcal{N}\left(\begin{array}{c}
m\left(X_{t_{i-1}}^{\prime}\right) \\
m\left(X_{t_{i-1}}^{*}\right)
\end{array},\left[\begin{array}{cc}
\mathcal{K}\left(X_{t_{i-1}}^{\prime}, X_{t_{i-1}}^{\prime T}\right) & \mathcal{K}\left(X_{t_{i-1}}^{\prime}, X_{t_{i-1}}^{* T}\right) \\
\mathcal{K}\left(X_{t_{i-1}}^{*}, X_{t_{i-1}}^{\prime T}\right) & \mathcal{K}\left(X_{t_{i-1}}^{*}, X_{t_{i-1}}^{* T}\right)
\end{array}\right]\right)
$$

A more detailed derivation on GPR is discussed by Rasmussen and Williams [14].

Employing the GPR method, we obtain an estimation of continuation value:

$$
\tilde{\mathrm{C}}_{t_{i-1}}=f\left(X_{t_{i-1}}\right)=\mathcal{G} \mathcal{P}_{t}\left(m\left(X_{t_{i-1}}\right), \mathcal{K}\left(X_{t_{i-1}}, X_{t_{i-1}}^{T}\right)\right)
$$

We can write $\tilde{\mathcal{C}}_{t_{i-1}}=\mathcal{P}_{t_{i-1}} V_{t_{i}}$ where $\mathcal{P}_{t_{i-1}}$ is the projection operator onto $\mathcal{F}_{t_{i-1}}$ measurable Gaussian Processes. We can also apply the learning stage on a smaller set than total amount of paths generated because GPR is computationally heavy with $O\left(M^{3}\right)$ operations.

Lastly, we improve the Kriging approach by including control variates (CV) in similar fashion as Rasmussen N. S. [13]. We use European put options control variates $Y_{T}=h\left(X_{T}\right)$ with maturity time $T=t_{N}$ written on the same underlying stochastic process and apply it directly to continuation value. In terms Gaussian process regression, we adjust the continuation value with the projection operator $\mathcal{P}_{t}$, in the following way:

$$
\mathcal{C}_{t_{i-1}}^{C V}=\mathcal{P}_{t_{i-1}} V_{t_{i}}-\tilde{\nu}_{t_{i-1}}\left(\mathcal{P}_{t-1} Y_{t_{i}}-\mathbb{E}\left[h\left(X_{t_{N}}\right) \mid X_{t_{i-1}}\right]\right)
$$

where $\tilde{\nu}_{t_{i-1}}$ minimizes the variance of $\mathcal{C}_{t_{i-1}}^{C V}$ :

$$
\tilde{\nu}_{t_{i-1}}=\frac{\mathcal{P}_{t_{i-1}}\left(V_{t_{i}} Y_{t_{i}}\right)-\mathcal{P}_{t_{i-1}} V_{t_{i}} \cdot \mathcal{P}_{t_{i-1}} Y_{t_{i}}}{\mathcal{P}_{t_{i-1}}\left(Y_{t_{i}}^{2}\right)-\left(\mathcal{P}_{t_{i-1}} Y_{t_{i}}\right)^{2}}
$$

We calculate the discounted price of European option $p_{t_{i-1}}=\mathbb{E}\left[h\left(X_{t_{N}}\right) \mid X_{t_{i-1}}\right]$ at each path via fast Fourier transform method described by Carr and Madan [2]. At each step, we adjust the control variate as $Y_{t_{N}}=h\left(X_{t_{N}}\right) Y_{t_{i-1}}=p_{t_{i-1}}$ if $h\left(X_{t_{i-1}}\right)>\mathcal{C}_{t_{i-1}}^{C V}$ otherwise $Y_{t_{i-1}}=Y_{t_{i}}$. At the last step, the control variate $Y_{t_{0}}$ is applied directly to the value of the option $V_{t_{0}}$.

\section{Numerical Study}

We design the numerical test to compare the three mentioned algorithms: LSM, Kriging and Kriging with CV over a set of different parameters. In the first part, we use standard examples and benchmarks from literature [12] to test the algorithms' efficiency. In the second part, we compare the methods on realistic parameters by first calibrating the Heston model to the market data from CBOE on Russell 2000 Index Options (RUT) and S\&P 500 Index Options (SPX). 


\subsection{Synthetic Data Experiment}

Let $t_{0}=0, t_{1}, \ldots, t_{N}=T$ be the equidistant discretization of the interval $[0, T]$ with the difference $\Delta t=\frac{T}{N}$. Then for $i=1,2, \ldots, N$, we can rewrite the discrete risk-neutral Heston dynamics (1) for sufficiently small $\Delta t$ :

$$
\begin{aligned}
\log S_{t_{i}} & =\log S_{t_{i-1}}+\left(r-\frac{1}{2} v_{t_{i-1}}\right) \Delta t+\sqrt{v_{t_{i-1}}} \sqrt{\Delta t} \epsilon_{S, t_{i}} \\
v_{t_{i}} & =v_{t_{i-1}}+\kappa^{*}\left(\theta^{*}-v_{t_{i-1}}\right) \Delta t+\sigma \sqrt{v_{t_{i-1}}} \sqrt{\Delta t}\left(\rho \epsilon_{S, t_{i}}+\sqrt{1-\rho^{2}} \epsilon_{v, t_{i}}\right)
\end{aligned}
$$

where $\left\{\epsilon_{S, t_{i}}, \epsilon_{v, t_{i}}\right\}_{i=1, \ldots, N}$ are i.i.d. random variables with standard normal distribution. This discretization method is also known as Euler-Maruyama. We replicate the correlation $<d W_{1, t}^{Q}, d W_{2, t}^{Q}>=\rho$ by constructing the random variable $<$ $\epsilon_{S, t}, \rho \epsilon_{S, t}+\sqrt{1-\rho^{2}} \epsilon_{v, t}>=\rho$ which has the same correlation and behavior. We replace the terms $v_{t_{i-1}}$ with $\max \left(v_{t_{i-1}}, 0\right)$ due to the fact that $v_{t_{i}}$ in discrete approximation may reach negative values for some extreme states of volatility.

For the three methods, we use backward dynamic programming formulation in (4) but we update the option value function:

$$
V_{t_{i-1}}= \begin{cases}h\left(S_{t_{i-1}}\right) & \text { if } h\left(S_{t_{i-1}}\right) \geq C_{t_{i-1}} \\ V_{t_{i}} & \text { otherwise }\end{cases}
$$

In this way, we don't add the errors from the estimation of continuation value in the option value function calculations.

In the LSM algorithm, we estimate the continuation value $\mathcal{C}$ as in equation (9) with the following basis functions in terms of stock prices $S$ and volatility $v$ :

$$
\Phi(S, v)=\left\{1, S, v, S v, S^{2}, v^{2}, S v, S^{3}, v^{3}, S^{2} v, S v^{2}\right\} .
$$

The interaction terms between $S$ and $v$ are necessary to capture the correlation $\rho$ of the Brownian motions of the stock price and its volatility. For the Kriging algorithm, we are estimating the continuation value $\mathcal{C}$ via Gaussian process regression (16) on the basis function $\{S, v\}$ and a random learning subset of paths of size $M_{0}$. Whereas, in the Kriging with $\mathrm{CV}$ we adjusting previous continuation value $\mathcal{C}$ as in equation (17), performing the other 3 GPR on the same set of basis functions.

In Table 1, we present the results for $S_{0} \in\{90,100,110\}, T \in\{0.25,0.5\}$ and $v_{0} \in\{0.04,0.09,0.16\}$ to account for in the money (ITM), at the money (ATM) and out of the money options (OTM) options with different maturities $T$ and riskiness $v_{0}$. For all of the test, GPR algorithm approximates the American options prices with a precision of up to 3 fold than LSM. With the addition of control variate, GPR algorithm further improves the precision (up to 10 fold) especially for high volatility, OTM options and high maturity. In Figure 1, we show that all 3 algorithm converge slowly as the number of simulated paths $M$ increases with $O(\sqrt{M})$ speed. The GPRCV algorithm reaches a suitable error even with $M$ as low as 2000. This showcases that Kriging with CV algorithm needs little information to output high precision results. 


\begin{tabular}{|lll|c|ccc|cccc|ccc|}
\hline & & & \multicolumn{4}{|c|}{ LSM } & \multicolumn{4}{c|}{ GPR } & \multicolumn{4}{c|}{ GPR-CV } \\
\multicolumn{1}{|c|}{$S_{0}$} & $v_{0}$ & $\mathrm{~T}$ & PSOR & \multicolumn{1}{|c|}{ Price } & Error \% & Std & Price & Error $\%$ & Std & Price & Error \% & Std \\
\hline \hline 90 & 0.04 & 0.25 & 10.1229 & 10.1653 & 0.4181 & 4.5720 & 10.1513 & 0.2806 & 4.3806 & 10.0990 & 0.2362 & 0.2808 \\
100 & 0.04 & 0.25 & 3.4813 & 3.5240 & 1.2258 & 4.1948 & 3.5068 & 0.7329 & 4.2624 & 3.4632 & 0.5212 & 0.1663 \\
110 & 0.04 & 0.25 & 0.8417 & 0.8741 & 3.8418 & 2.4929 & 0.8735 & 3.7729 & 2.4695 & 0.8282 & 1.6069 & 0.0929 \\
\hline 90 & 0.09 & 0.25 & 10.9573 & 11.0423 & 0.7756 & 7.0613 & 11.0121 & 0.4997 & 6.8796 & 10.9374 & 0.1818 & 0.2927 \\
100 & 0.09 & 0.25 & 4.9461 & 5.0244 & 1.5824 & 6.3102 & 4.9920 & 0.9284 & 6.0819 & 4.9339 & 0.2479 & 0.1751 \\
110 & 0.09 & 0.25 & 1.8641 & 1.9244 & 3.2331 & 4.2084 & 1.9068 & 2.2883 & 4.1085 & 1.8581 & 0.3249 & 0.0918 \\
\hline 90 & 0.16 & 0.25 & 12.1200 & 12.2376 & 0.9703 & 9.1855 & 12.1845 & 0.5320 & 8.8658 & 12.1059 & 0.1163 & 0.2680 \\
100 & 0.16 & 0.25 & 6.4933 & 6.6016 & 1.6676 & 8.0982 & 6.5553 & 0.9551 & 7.8332 & 6.4841 & 0.1409 & 0.1753 \\
110 & 0.16 & 0.25 & 3.1470 & 3.2385 & 2.9067 & 6.0491 & 3.2003 & 1.6923 & 5.8564 & 3.1444 & 0.0820 & 0.1061 \\
\hline 90 & 0.04 & 0.5 & 10.5667 & 10.6314 & 0.6127 & 5.9602 & 10.6110 & 0.4198 & 5.8109 & 10.5373 & 0.2782 & 0.5463 \\
100 & 0.04 & 0.5 & 4.6645 & 4.7266 & 1.3311 & 5.9187 & 4.6846 & 0.4295 & 5.6213 & 4.6460 & 0.3963 & 0.3512 \\
110 & 0.04 & 0.5 & 1.7875 & 1.8421 & 3.0558 & 4.0904 & 1.8262 & 2.1650 & 3.9732 & 1.7741 & 0.7504 & 0.1807 \\
\hline 90 & 0.09 & 0.5 & 11.7658 & 11.8552 & 0.7595 & 8.3687 & 11.8166 & 0.4318 & 8.0841 & 11.7463 & 0.1654 & 0.5730 \\
100 & 0.09 & 0.5 & 6.2573 & 6.3427 & 1.3652 & 7.6451 & 6.2935 & 0.5799 & 7.3566 & 6.2435 & 0.2195 & 0.4012 \\
110 & 0.09 & 0.5 & 3.0673 & 3.1497 & 2.6870 & 5.8725 & 3.1049 & 1.2273 & 5.6399 & 3.0602 & 0.2318 & 0.2432 \\
\hline 90 & 0.16 & 0.5 & 13.2329 & 13.3473 & 0.8642 & 10.4700 & 13.2823 & 0.3733 & 10.0642 & 13.2159 & 0.1285 & 0.5669 \\
100 & 0.16 & 0.5 & 8.0073 & 8.1207 & 1.4158 & 9.5221 & 8.0532 & 0.5739 & 9.1627 & 7.9973 & 0.1243 & 0.4255 \\
110 & 0.16 & 0.5 & 4.6232 & 4.7312 & 2.3360 & 7.8025 & 4.6693 & 0.9967 & 7.4842 & 4.6194 & 0.0829 & 0.2936 \\
\hline
\end{tabular}

Table 1. Convergence of American put option prices computed by Least Square Method, Gaussian process regression with/without control variate averaged over 100 independent runs versus PSOR Benchmark. Parameters: $K=100, r=0.05, \kappa=3$, $\theta=0.04, \sigma=0.1, \rho=-0.7, N=100, M=5000$ and $M_{0}=2000$

\subsection{Market Data Experiment}

We aim to price American options on RUT and SPX indexes with LSM and GPRCV. We select European put and call option prices of the 2 indexes at "24-08-2015" having stock prices $S_{R U T}=1111.69$ and $S_{S P X}=1893.2$, with maturity "19-12-2015" $(\mathrm{T}=0.32)$ and "18-03-2016" $(\mathrm{T}=0.57)$ and with strike prices $K$ in range of $10 \%$ of the stock price.

For both indices, we derive the dividend yield $q$ and interest rate $r$ using Put-Call parity on the market data prices via a linear regression as in Ackerer [1]:

$$
C_{m k t}-P_{m k t}=e^{-q T} S_{0}-e^{-r T} K .
$$

where $C_{m k t}, P_{m k t}$ are the market prices of the call and put with strike price $K$ and maturity $T$ on index $S_{0}$. We calibrate the parameters of the Heston model by minimizing the mean squared distance between the Black-Scholes implied volatility of market option prices and Heston option prices [1]:

$$
\min _{\left\{\sigma, v_{0}, \kappa, \theta, \rho\right\}} \sqrt{\frac{1}{|\mathbb{O}|} \sum_{o \in \mathbb{O}}\left(\sigma_{m k t}^{i m p}-\sigma_{\text {Heston }}^{i m p}\right)^{2}}
$$

where $\mathbb{O}$ are the set of options on the same index, $\sigma_{m k t}^{i m p}$ is the implied volatility of market price of the option and $\sigma_{\text {Heston }}^{i m p}$ is the implied volatility of the option price 


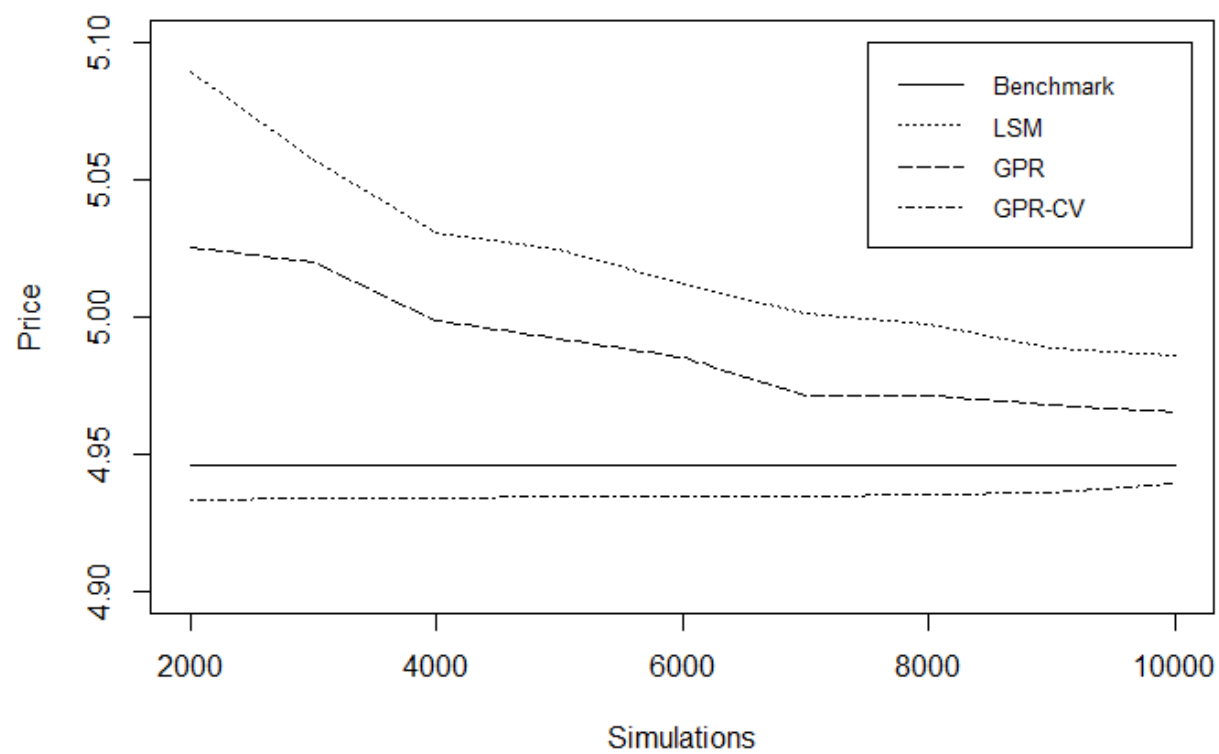

Figure 1. American put option price behavior averaged over 100 independent runs. Parameters: $S_{0}=100, K=100, T=0.25, v_{0}=0.09, r=0.05, \kappa=3, \theta=0.04$, $\sigma=0.1, \rho=-0.7, N=100$ and $M_{0}=2000$

calculated via Car-Madan formula on Heston model. As a result, we obtain the following parameters:

\begin{tabular}{|c|c|c|c|c|c|c|c|c|}
\hline Index & $r$ & $q$ & $r-q$ & $\sigma$ & $v_{0}$ & $\kappa$ & $\theta$ & $\rho$ \\
\hline \hline RUT & 0.0229 & 0.0057 & 0.0171 & 0.8669 & 0.1098 & 6.4541 & 0.0487 & -0.5751 \\
\hline SPX & 0.0211 & 0.0060 & 0.0151 & 0.8497 & 0.1070 & 6.6356 & 0.0302 & -0.5630 \\
\hline
\end{tabular}

Table 2. Calibrated Parameters

With the calibrated parameters, we apply the same procedure as in Section 3.1 to price the American options on RUT and SPX indices and report the results in the Table 3. We arrive at the same conclusion that GPR-CV algorithm outperforms LSM algorithm especially for OTM options.

\section{Conclusions}

We have laid down the backward dynamic principle for American options pricing in the context of Monte Carlo methods. We have presented the LSM and Kriging approach in 


\begin{tabular}{|c|cc|c|ccc|ccc|}
\hline \multirow{2}{*}{ Index } & & & & \multicolumn{3}{|c|}{ LSM } & \multicolumn{3}{c|}{ GPR-CV } \\
& $\mathrm{K}$ & $\mathrm{T}$ & PSOR* & Price & Error \% & Std & Price & Error \% & Std \\
\hline \hline \multirow{6}{*}{ RUT } & 1000 & 0.32 & 25.396 & 26.443 & 4.1219 & 62.190 & 25.411 & 0.0606 & 1.111 \\
& 1110 & 0.32 & 61.684 & 63.057 & 2.2260 & 91.084 & 60.892 & 1.2830 & 2.989 \\
& 1220 & 0.32 & 127.339 & 129.190 & 1.4535 & 110.985 & 125.774 & 1.2292 & 3.624 \\
& 1000 & 0.57 & 36.524 & 37.902 & 3.7715 & 78.755 & 36.484 & 0.1114 & 1.862 \\
& 1110 & 0.57 & 75.654 & 77.315 & 2.1956 & 107.009 & 74.502 & 1.5230 & 4.028 \\
& 1220 & 0.57 & 138.775 & 140.580 & 1.3003 & 126.566 & 136.353 & 1.0245 & 5.352 \\
\hline \multirow{5}{*}{ SPX } & 1700 & 0.32 & 35.983 & 37.668 & 4.6825 & 95.029 & 36.013 & 0.0845 & 1.868 \\
& 1900 & 0.32 & 99.744 & 102.115 & 2.3763 & 146.753 & 98.129 & 1.6194 & 5.538 \\
& 2100 & 0.32 & 226.829 & 229.756 & 1.2905 & 176.642 & 224.018 & 1.2390 & 6.578 \\
& 1700 & 0.57 & 49.137 & 51.341 & 4.4850 & 117.266 & 49.105 & 0.0651 & 3.164 \\
& 1900 & 0.57 & 116.784 & 119.359 & 2.2041 & 166.954 & 114.949 & 1.5714 & 7.759 \\
& 2100 & 0.57 & 237.415 & 241.384 & 1.6720 & 196.110 & 234.265 & 1.3269 & 9.638 \\
\hline
\end{tabular}

Table 3. Performance of LSM, GPR-CV on RUT and SPX versus PSOR benchmark. Parameters from Table 2 and $N=100, M=5000, M_{0}=2000$.

* American Option price calculated via explicit scheme with discretization $\Delta_{S}=2^{10}, \Delta_{v}=2^{9}$ and $\Delta_{T}=2^{20}$.

this framework together with control variate adjustment. In particular, we have shown that GPR method always outperforms LSM algorithm especially when combined with European counterpart as a control variate but at an additional computational cost. The numerical results on OTM options illustrate that GPR-CV is well equipped to deal with minimal amount of information and provide precise pricing results.

\section{References}

[1] Ackerer D. and Filipovic D. (2017), 'Option Pricing with Orthogonal Polynomial Expansions', Swiss Finance Institute Research Paper No. 17-41.

[2] Carr P. and Madan D. (1999), 'Option valuation using the fast Fourier transform', New York, NY: John Wiley \& Sons.

[3] Glasserman P. (2003), 'Monte Carlo Methods in Financial Engineering', Springer Science and Business Media New York.

[4] Glasserman P. and Yu B. (2004). 'Number of paths versus basis functions in American option pricing.' The Annals of Applied Probability, 14(4):2090-2119.

[5] Gramacy R. and Ludkovski M. (2015), 'Sequential design for optimal stopping problems', SIAM Journal on Financial Mathematics, p. 748-775.

[6] Heston S., (1993), 'A closed-form solution for options with stochastic volatility with applications to bond and currency options', Review of Financial Studies.

[7] Li Y., Szepesvari C. and Schuurmans D. (2008), Learning an Exercise Policy for American Options', Proceedings of the $12^{\text {th }}$ AISTATS. 
[8] Longstaff F. and Schwartz E. (2001), 'Valuing American options by simulations: a simple least squares approach', The Review of Financial Studies, 14 , pp. 113148.

[9] Kloeden P. and Platen E. (1992), 'Numerical Solution of Stochastic Differential Equations'. Berlin: Springer-Verlag.

[10] Ludkovski M. (2016), 'Kriging Metamodels and Experimental Design for Bermudan Option Pricing', arXiv preprint arXiv:1509.02179, pp. 748-775.

[11] Pizzi C. and Pellizzari P. (2002) 'Monte Carlo Pricing of American Options Using Nonparametric Regression', Rendiconti per gli Studi Economici Quantitativi, 7591.

[12] O'Sullivan C. and O'Sullivan S. (2013) 'Pricing European and American Options in the Heston Model with Accelerated Explicit Finite Differencing Methods', International Journal of Theoretical and Applied Finance Vol. 16, No. 3

[13] Rasmussen N. S. (2005) 'Control variates for Monte Carlo valuation of American options', Journal of Computational Finance, vol. 9, no. 1, 09.

[14] Rasmussen C. E. and Williams C. K. I. (2006), 'Gaussian Processes for Machine Learning', MIT Press

[15] Tsitsiklis J. and Van Roy B. (2001), 'Regression methods for pricing complex American-style options', IEEE Transactions on Neural Networks, 12 , pp. 694703.

Received 02.02.2018, Accepted 05.09.2018 